Received: 27.11 .2018

Revised: 18.12 .2018

Accepted: 21.12 .2018

DOI: $10.17804 / 2410-9908.2018 .6 .222-228$

\title{
MICROMAGNETIC MODELING OF MAGNETIZATION AUTORESONANCE IN YIG THIN FILMS WITH INDUCED UNIAXIAL ANISOTROPY
}

\author{
V. S. Teplov ${ }^{\text {a) }}$ and V. D. Bessonov ${ }^{\text {b)* }}$ \\ M.N. Miheev Institute of Metal Physics, UB of RAS, \\ 18 S. Kovalevskoy St., Ekaterinburg, 620137, Russian Federation \\ a) iD https://orcid.org/0000-0002-3986-4768 st.valintaine@gmail.com ; \\ b) iD https://orcid.org/0000-0002-7837-4366 bessonov@imp.uran.ru \\ *Corresponding author E-mail: bessonov@imp.uran.ru \\ Address for correspondence: ul. S. Kovalevskoy, 18, Ekaterinburg, 620137, Russian Federation \\ Tel.: +7 (343) 37837 43; fax: +7 (343) 3745244
}

One of the main tasks of both magnonics and physics of nonlinear processes on the search for ways of exciting efficiently magnetic oscillations in solids is discussed. According to the earlier developed theoretical predictions, the MuMax3 software is used to carry out a micromagnetic simulation of a nonlinear effect of strong autoresonance amplifying of the amplitude of magnetic oscillations in yttrium-iron garnet under the action of an external excitation field. It is shown that the efficiency of this autoresonance excitation in thin films of ferrimagnetic yttrium-iron garnet nonlinearly depends on the power of the exciting field and the rate of change (scanning) of the exciting frequency. Both the rate of change and power of the exciting frequency are estimated with the aim of direct experimental observation of the autoresonance effect, in view of the real parameters of the yttrium-iron garnet film at room temperature.

Keywords: autoresonance, spin waves, spin dynamics, micromagnetic simulation, YIG.

\section{Acknowledgment}

We are grateful to Dr. S. V. Batalov and Dr. A. V. Telegin for their assistance in the preparation of the paper.

The work was performed within the state assignment from FASO Russia on the subject of Spin, No. AAAA-A18-118020290104-2, and partially supported by megagrant No. 14.Z50.31.0025 and grant No. MK-4959.2018.2 from the President of Russia for young scientists.

\section{References}

1. Demidov V.E., Kostylev M.P., Rott K., Krzysteczko P., Reiss G., Demokritov S.O. Excitation of microwave guide modes by a stripe antenna. Applied Physics Letters, 2009, vol. 95, no. 11, pp. 112509. DOI: https://doi.org/10.1063/1.3231875.

2. Kajiwara Y., Harii K., Takahashi S., Ohe J., Uchida K., Mizuguchi M., Umezawa H., Kawai H., Ando K., Takanashi K., Maekawa S., Saitoh E. Transmission of electrical signal by spin-waves interconversion in a magnetic insulator. Nature, 2010, vol. 464, pp. 262-266. DOI: 10.1038/nature08876.

3. Batalov S.V., Shagalov A.G. Autoresonance control of a magnetization soliton. The Physics of Metals and Metallography, 2010, vol. 109, no. 1, pp. 1-6. DOI: 10.1134/S0031918X10010011.

4. Batalov S.V., Shagalov A.G. Resonance control of solitons of spin waves. The Physics of Metals and Metallography, 2013, vol. 114, no. 10, pp. 826-832. DOI: https://doi.org/10.1134/ S0031918X13100025. 
5. Shamsutdinov M.A., Kalyakin L.A., Kharisov A.T. Autoresonance in a ferromagnetic film. Technical Physics, 2010, vol. 55, no. 6, pp. 860-865. DOI: https://doi.org/10.1134/S1063784210060198.

6. Vansteenkiste A., Leliaert J., Dvornik M., Helsen M., Garcia-Sanchez F. The design and verification of MuMax3. In: AIP Advances, 2014, vol. 4, pp. 107133. DOI: 10.1063/1.4899186.

7. Bokov V.A. Fizika magnetikov [Physics of Magnetics]. Nevskiy Dialekt Publ., 2002, 143 p. (In Russian).

8. Hurben M.J., Patton C. Theory of magnetostatic waves for in-plane magnetized anisotropic films. Journal of Magnetism and Magnetic Materials, 1996, vol. 163, pp. 39-69. DOI: 10.1016/03048853(95)90006-3. 
Подана в журнал: 27.11.2018

УДК 537.635

DOI: $10.17804 / 2410-9908.2018 .6 .222-228$

\title{
МИКРОМАГНИТНОЕ МОДЕЛИРОВАНИЕ ЯВЛЕНИЙ АВТОРЕЗОНАНСА НАМАГНИЧЕННОСТИ В ТОНКИХ ПЛЕНКАХ ЖЕЛЕЗО-ИТТРИЕВОГО ГРАНАТА С НАВЕДЕННОЙ ОДНООСЕВОЙ АНИЗОТРОПИЕЙ
}

\author{
В. С. Теплов ${ }^{\text {a) }}$ В. Д. Бессонов ${ }^{\text {б)* }}$ \\ Институт Физики Металлов имени М.Н. Михеева Уральского отделения Российской академии наук, \\ ул. С. Ковалевской 18, Екатеринбург, Российская Федераџия \\ a) iD https://orcid.org/0000-0002-3986-4768 st.valintaine@gmail.com ; \\ б) (iD https://orcid.org/0000-0002-7837-4366 bessonov@imp.uran.ru \\ *Ответственный автор. Электронная почта: bessonov@imp.uran.ru \\ Адрес для переписки: 620137, Екатеринбург, ул. С. Ковалевской, 18, Российская Федерация \\ Тел.: +7 (343) 37837 43; факс: +7 (343) 3745244
}

В работе рассматривается одна из основных задач магноники и физики нелинейных процессов по поиску путей эффективного возбуждения спиновых волн в магнетике. Используя развитую теоретическую модель авторезонансных колебаний в магнетиках, в программной среде МuMaх3 впервые проведено микромагнитное моделирование нелинейного эффекта автоусиления амплитуды осцилляций магнитной подсистемы в железоиттриевом гранате под действием переменного возбуждающего поля. Показано, что эффективность возбуждения авторезонанса в тонких пленках ферримагнитного железоиттриевого граната нелинейно зависит от мощности возбуждающего поля и скорости изменения возбуждающей частоты. Оценены необходимые параметры скорости изменения и мощности возбуждающей частоты для наблюдения авторезонанса в эксперименте с учетом реальных параметров пленки железоиттриевого граната при комнатной температуре.

Ключевые слова: авторезонанс, спиновые волны, спиновая динамика, микромагнитное моделирование, ЖИГ.

\section{1. Введение}

Одной из фундаментальных задач теории магнитных явлений и нелинейной динамики намагниченности является поиск путей эффективного возбуждения и управления распространением высокочастотных (CBЧ) магнитных колебаний в среде. На данный момент такие процессы очень энергозатратны и требуют мощного источника СВЧ-тока, подаваемого на возбуждающую антенну [1], либо спиновый инжектор [2]. В работе [3] впервые теоретически было показано, что магнитные колебания в магнетиках можно возбуждать с помощью нелинейного эффекта авторезонанса. В сравнении с обычным частотным резонансом, в данном случае резонансное состояние достигается малыми (порядка доли эрстед) магнитными полями, при использовании эффекта синхронизации фаз возбуждающего поля и возбужденной волны. Для этого с заданной скоростью модулируется частота внешнего возбуждающего переменного магнитного поля, при этом возбуждаемые колебания в твердом теле должны возникать на той же частоте, независимо от собственного спектра. Также этот процесс можно использовать для эффективного возбуждения и исследования различных солитонных состояний [3-5].

Данный эффект является новым и экспериментально не наблюдался. К сожалению, развитые аналитические модели авторезонансного эффекта в магнетиках используют много приближений. Например, не учитывается геометрия образца (размагничивающий фактор), 
коэффициенты затухания, диполь-дипольное взаимодействие магнитных моментов и т. д. В результате сложно спрогнозировать обнаружение этого эффекта на практике, например, в часто используемых в СВЧ-электронике пленочных магнетиках с малой величиной затухания СВЧ-сигнала типа железо-иттриевый гранат (ЖИГ - $\mathrm{Y}_{3} \mathrm{Fe}_{5} \mathrm{O}_{12}$ ).

Одним из эффективных теоретических методов решения задач возбуждения и распространения спиновых волн в магнетике с учетом реальных граничных условий является микромагнитное моделирование [6]. В работе проведено микромагнитное моделирование явления нелинейного эффекта усиления амплитуды осцилляций магнитной подсистемы в тонких пленках ЖИГ. Полученные данные хорошо согласуются с результатами аналитической теории [3]. Результаты работы могут быть использованы для постановки эксперимента на пленках ЖИГ и проверки теории.

\section{2. Постановка задачи и методы решения}

Микромагнитное моделирование было выполнено в программной оболочке MuMax3 [6]. В качестве объекта моделирования была выбрана пленка ЖИГ, поскольку данный материал обладает максимально низким уровнем затухания спиновой подсистемы, что обеспечивает устойчивость линейных и нелинейных волновых процессов.

В качестве модельной единицы был выбран единичный объем магнетика в виде квадрата размером $1500 \times 1500 \times 150$ нм$^{3}$. Пленка ЖИГ была смоделирована в виде матрицы из элементарных ячеек размером $30 \times 30 \times 15$ нм$^{3}$. Взаимодействия между ячейками рассчитывали с помощью уравнения Ландау-Лифшица [5]. В качестве материальных констант были выбраны следующие параметры для ЖИГ: $M_{s}=139261 \mathrm{~A} / \mathrm{M}$ - намагниченность насыщения; $\alpha_{G}=0,0001$ - константа затухания Гилберта; $A_{e x}=4,15 \times 10^{-12}$ Дж/м - константа неоднородного обмена [7]. Толщина пленки подбиралась из условия кратности количества магнитных длин $l=\sqrt{A_{e x} / k_{u}}$ десяти. Вклад магнитокристаллической анизотропии в плоскости пленки ввиду его малости не учитывался [8].

Для сопоставления результатов моделирования с аналитическим решением задачи управления нелинейной волной намагниченности при помощи внешнего переменного магнитного поля в магнетике с анизотропией типа - ось легкого намагничивания [3], в модели пленки ЖИГ дополнительно была учтена одноосевая анизотропия $\left(k_{u}\right)$ в направлении перпендикулярной плоскости пленки $\left(k_{u}=13155\right.$ Дж/ $\mathrm{m}^{3}-$ константа одноосной анизотропии, такая, чтобы нормализованная константа анизотропии $\left.\beta=k_{u} / 2 M_{s}=1\right)$. Диполь-дипольное взаимодействие магнитных моментов между ячейками также не учитывалось для простоты моделирования.

\section{3. Результаты и обсуждения}

В выбранной модели пленка ЖИГ намагничивалась в нормальном направлении относительно поверхности в поле $H_{z}=0,35$ Тл. Для определения спектра собственных колебаний в плоскости пленки прикладывалось переменное поле $H_{x}$ :

$$
H_{x}=h_{0} \frac{\sin \left(f_{0}\left(t-t_{0}\right)\right)}{f_{0}\left(t-t_{0}\right)}
$$

где $h_{0}=10^{-3}$ Тл; $f_{0}=40$ ГГц, $t_{0}=0,1$ нс. Спектр такой функции имеет постоянную амплитуду до частоты $f_{0}$, после чего она резко спадает до нуля.

После возбуждения импульса фиксировалось поведение намагниченности от времени в течение 100 нс. Затем массив полученных данных подвергался Фурье преобразованию и в результате был получен спектр магнитных колебаний для образца пленки ЖИГ (рисунок). Спектр представляет собой набор дискретных мод. Для характеризации этих мод изучалось распределение динамической намагниченности по толщине образца. Из этих данных определена частота 
ферромагнитного резонанса $\omega_{0}=14,1$ ГГц как первой моды, где наблюдается стоячая волна с гармоническим распределением с узлами на поверхностях образца. Также была определена частота первой моды спин-волнового резонанса $\omega_{1}=15,4$ ГГц как стоячей волны с гармоническим распределением и узлами на поверхностях образца и перегибом по центру пленки. Интенсивная высокочастотная мода связана с ограничением возможных возбуждаемых длин волн размерами элементарной ячейки и является артефактом численного счета. Можно отметить, что увеличение толщины пленки ЖИГ приведет к перераспределению наблюдаемых мод за счет включения диполь-дипольного взаимодействия, это в данной работе не рассматривалось.

Эффект авторезонанса проявляется в области ниже спектра собственных магнитных колебаний системы (ФМР). Для возбуждения эффекта необходимо линейно уменьшать частоту начиная от первой моды спин-волнового резонанса и далее через ферромагнитный резонанс в область частот, где отсутствуют собственные магнитные колебания образца. При этом величина вектора динамической намагниченности должна возрастать. Магнитные колебания в пленке ЖИГ возбуждаются линейным возбуждающим переменным гармоническим полем $h_{0}$ с амплитудами $10^{-5}$ и $10^{-3}$ Тл, направленным в плоскости пленки нормально к постоянному полю намагничивания $H_{z}$. Возбуждающая частота меняется по линейному закону:

$$
\omega(t) \sim \omega_{\text {start }}+\frac{\varepsilon \beta^{2} \omega_{0}^{2}}{2} t
$$

где $\omega_{\text {start }}-$ начальная частота, выбирается как первая мода спин-волнового резонанса, а $\omega_{\text {res }}-$ частота ферромагнитного резонанса, определяемая как наименьший пик в спектре магнитных колебаний, полученных из моделирования; $\beta=k_{u} / 2 M_{s}-$ нормированная константа одноосевой анизотропии; $\varepsilon$ - константа пропорциональности, характеризующая скорость изменения частоты $\varepsilon \approx 10^{-6}$ рад $^{-1}$.

В качестве начальной частоты была взята $\omega_{\text {start }}=18$ ГГц и для ферромагнитного резонанса $\omega_{0}=14,1$ ГГц. Затем для данных частот подбиралась оптимальная скорость изменения (сканирования) частоты возбуждающего гармонического линейного поля.

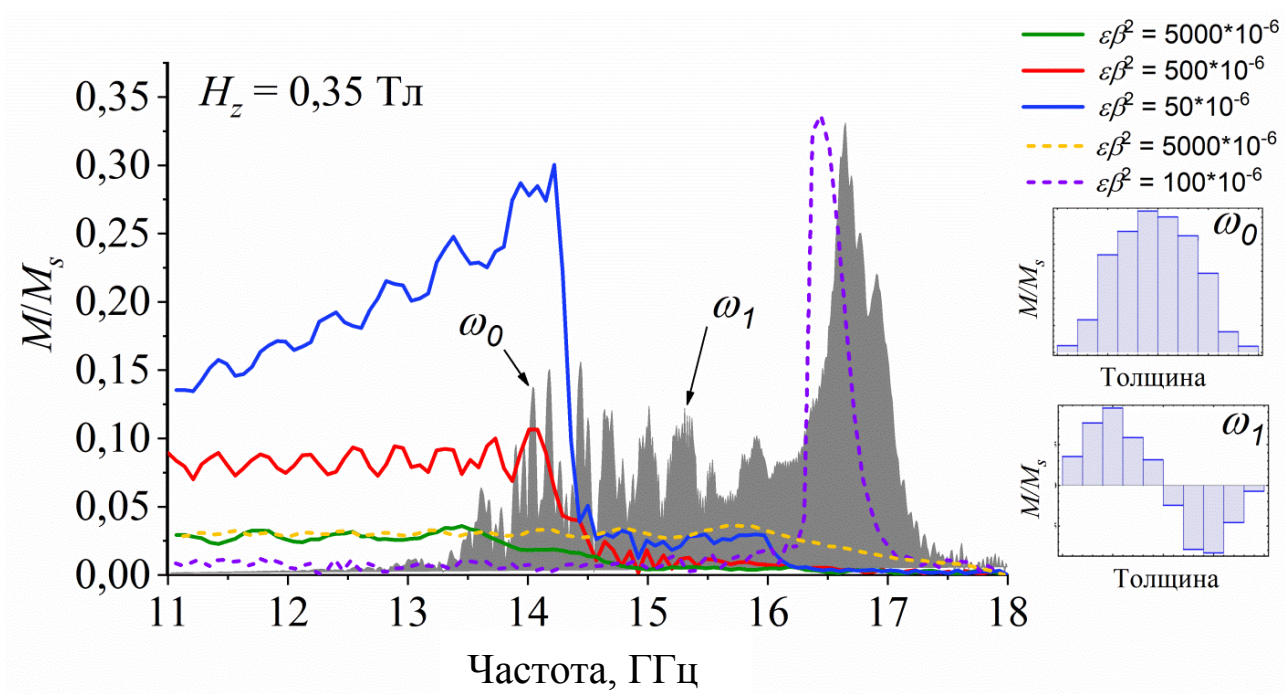

Расчетные спектры колебаний динамической намагниченности в пленке ЖИГ для разных скоростей сканирования $\varepsilon \beta^{2}$. Серый фон: спектр собственных колебаний образца пленки ЖИГ. Линиями показаны интенсивности динамической намагниченности при возбуждении авторезонанса в поле $10^{-5}$ Тл (сплошная линия) и $10^{-3}$ Тл (пунктирная линия).

На вставке справа - распределение амплитуды динамической намагниченности по толщине пленки для пиков $\omega_{0}$ и $\omega_{1}$ 
Особенностью спектров колебаний динамической намагниченности в пленке ЖИГ является зависимость от мощности возбуждающего поля, заданного по уравнению (2), и скорости изменения (сканирования) возбуждающей частоты. Таким образом, наблюдается проявление авторезонанса как это было предсказано теорией в [3]. Для минимальной скорости сканирования $\varepsilon \beta^{2}=50 \times 10^{-6}$ в направлении от максимальных к минимальным частотам наблюдается резкое увеличение амплитуды колебаний динамической намагниченности (до 30 \%) на частоте 14,4 ГГц вблизи ферромагнитного резонанса, связанное с проявлением эффекта авторезонанса, затем амплитуда постепенно спадает (рисунок), но имеются ненулевые значения в области ниже 13,6 ГГц, где отсутствуют собственные колебания намагниченности. Увеличение скорости сканирования до $\varepsilon \beta^{2}=500 \times 10^{-6}$ приводит практически к частотнонезависимому виду спектра колебаний, даже при достаточно высоких амплитудах динамической намагниченности. Дальнейшее увеличение скорости до $\varepsilon \beta^{2}=5000 \times 10^{-6}$ сканирования приводит к уменьшению амплитуды динамической намагниченности. При всех скоростях сканирования наблюдаются слабые осцилляции амплитуды динамической намагниченности, аналогичные наблюдаемым в статье [3].

Увеличение амплитуды возбуждающего поля до $10^{-3}$ Тл резко меняет спектральное поведение авторезонанса. При малых скоростях сканирования до $\varepsilon \beta^{2}=100 \times 10^{-6}$ имеется резкий максимум на артефактном пике, после чего амплитуда колебаний спадает почти до нуля. При увеличении скорости сканирования до $\varepsilon \beta^{2}=5000 \times 10^{-6}$ проявляется набор мод с колебаниями слабой интенсивности, которые почти не зависят от частоты сканирования и величины возбуждающего поля. Таким образом, можно предположить, что наблюдаемые в поле $10^{-3}$ Тл моды не связаны с авторезонансом.

\section{4. Заключение}

Таким образом, в работе методом микромагнитного моделирования впервые была выявлена возможность возбуждения авторезонанса в пленке ЖИГ за счет плавного изменения частоты возбуждающего поля. Показан нелинейный характер зависимости величины авторезонанса от скорости изменения и напряженности возбуждающего магнитного поля. Оценены параметры скорости изменения возбуждающей частоты для возбуждения авторезонанса и определены условия для экспериментального обнаружения эффекта в тонких пленках ЖИГ. Для эффективного возбуждения колебаний динамической намагниченности большой амплитуды в пленке ЖИГ толщиной 150 нм необходимо намагнитить пленку до насыщения в поле $H_{z}=0,35$ Тл по направлению, перпендикулярному поверхности пленки. Переменное возбуждающее магнитное поле необходимо менять со скоростью $\left(\varepsilon \beta^{2} \omega_{0}{ }^{2}\right) / 2 \approx 350$ МГц/с.

\section{Благодарность}

Авторы благодарны к.ф.-м.н. С.В. Баталову и к.ф.-м.н. А.В. Телегину за помощь в работе над статьей.

Работа выполнена в рамках государственного задания ФАНО России (тема «Спин» АААA-A18-118020290104-2), при частичной поддержке мегагранта №14.Z50.31.0025 и гранта Президента РФ для молодых ученых МК-4959.2018.2.

\section{Литература}

1. Excitation of microwave guide modes by a stripe antenna / V. E. Demidov, M. P. Kostylev, K. Rott, P. Krzysteczko, G. Reiss, S. O. Demokritov // Applied Physics Letters. - 2009. - Vol. 95, no. 11. - P. 112509. - DOI: https://doi.org/10.1063/1.3231875.

2. Transmission of electrical signal by spin-waves interconversion in a magnetic insulator / Y. Kajiwara, K. Harii, S. Takahashi, J. Ohe, K. Uchida, M. Mizuguchi, H. Umezawa, H. Kawai, 
K. Ando, K. Takanashi, S. Maekawa, E. Saitoh // Nature. - 2010. - Vol. 464. - P. 262-266. DOI: $10.1038 /$ nature08876.

3. Batalov S. V., Shagalov A. G. Autoresonance control of a magnetization soliton // The Physics of Metals and Metallography. - 2010. - Vol. 109, no. 1. - P. 1-6. DOI: https://doi.org/10.1134/S0031918X10010011.

4. Batalov S. V., Shagalov A. G. Resonance control of solitons of spin waves. // The Physics of Metals and Metallography. - 2013. - Vol. 114, no. 10. - P. 826-832. DOI: https://doi.org/10.1134/S0031918X13100025.

5. Shamsutdinov M. A., Kalyakin L. A., Kharisov A. T. Autoresonance in a ferromagnetic film // Technical Physics. - 2010. - Vol. 55, no. 6. - P. 860-865. DOI: https://doi.org/10.1134/S1063784210060198.

6. The design and verification of MuMax3 / A. Vansteenkiste, J. Leliaert, M. Dvornik, M. Helsen, F. Garcia-Sanchez // AIP Advances. - 2014. - Vol. 4. - P. 107133. DOI: https://doi.org/10.1063/1.4899186.

7. Боков В. А. Физика магнетиков. - Санкт-Петербург : Издательство «Невский диалект», 2002. - $143 \mathrm{c}$.

8. Hurben M. J., Patton C. E. Theory of magnetostatic waves for in-plane magnetized anisotropic films // Journal of Magnetism and Magnetic Materials. - 1996. - Vol. 163. - P. 39-69. DOI: 10.1016/0304-8853(95)90006-3. 